\title{
Qualidade de vida e funcionalidade nos pacientes com desordens do manguito rotador
}

\author{
Quality of life and functionality in patients with rotator \\ cuff disorders
}

\section{Ricardo de Almeida Albuquerque', Martha Moreira Cavalcante Castro², Marcos Antonio Almeida Matos ${ }^{3}$, Carla Hilario da Cunha Daltro ${ }^{4}$}

\begin{abstract}
'Autor para correspondência. Escola Bahiana de Medicina e Saúde Pública. Salvador, Bahia, Brasil. ORCID: 0000-0003-2960-9971.ric_fisio@ig.com.br ${ }^{2}$ Universidade Federal da Bahia. Salvador, Bahia, Brasil. ORCID: 0000-0002-7868-1120. marthamccastro@gmail.com ${ }^{3}$ Escola Bahiana de Medicina e Saúde Pública. Salvador, Bahia, Brasil. ORCID: 0000-0002-3592-986X. marcos.almeida@hotmail.com ${ }^{4}$ Universidade Federal da Bahia. Salvador, Bahia, Brasil. ORCID: 0000-0003-1115-688X. carlahcd@terra.com.br
\end{abstract}

RESUMO | OBJETIVO: Verificar se existe correlação entre os domínios da qualidade de vida e a funcionalidade nos pacientes com desordens do manguito rotador. MÉTODO: Estudo do tipo transversal com 109 pacientes avaliados entre outubro 2013 e fevereiro 2014. Para avaliar a qualidade de vida foi utilizado o questionário WORC, e as escalas UCLA e ASES para avaliação da funcionalidade. RESULTADO: Amostra de 73 mulheres e 36 homens (média de idade: 52,3 anos $+12,8$ ). $O$ ombro avaliado foi o direito em 63 pacientes $(57,8 \%)$. O WORC apresentou correlação positiva alta com as escalas UCLA $(r=0,73)$ e ASES $(r=0,77)$. Ao analisar o WORC verificou-se correlação mais forte com a UCLA no domínio "Trabalho" $(r=$ $0,74)$, e menor no domínio "Esporte/Recreação" $(r=0,67)$. Quando comparado o WORC com a ASES identificou-se a maior correlação com o domínio "Trabalho" $(r=0,79)$ e menor correlação com o domínio "Emoções" $(r=0,64)$. CONCLUSÃO: Alterações na funcionalidade, frequentes nestas desordens, impactam na qualidade de vida em especial no domínio "Trabalho".

PALAVRAS-CHAVE: Manguito rotador. Qualidade de vida. Questionários. Escalas funcionais.

\begin{abstract}
OBJECTIVE: To evaluate the correlation between different domains of quality of life and functionality in patients with rotator cuff disorders. METHODS: Cross sectional study with 109 patients evaluated between October 2013 and February 2014. The WORC questionnaire was used to evaluate quality of life, and the UCLA and ASES scales to evaluate functionality. RESULTS: The sample included 73 female and 36 males (mean age $52,3+12,8$ years). The right shoulder was evaluated in 63 patients $(57,8 \%)$. There was a high positive correlation between the WORC results and the UCLA $(r=0,73)$ and ASES $(r=0,77)$ scales. There was a stronger correlation between the WORC and the UCLA in the "Work" domain $(r=0,74)$, and a weaker correlation in the "Sport/Recreation" domain ( $r=0,67)$. When comparing the WORC with the ASES scale, a higher correlation was identified with the "Work" domain $(r=$ $0,79)$ and a lower correlation with the "Emotions" domain $(r=0,64)$. CONCLUSION: Alterations in functionality, frequent in RCD, have a considerable impact in the quality of life in special to the "Work" domain.
\end{abstract}

KEYWORDS: Rotator cuff. Quality of life. Questionnaires. Functional outcomes. 


\section{Introdução}

A dor no ombro é a terceira causa mais comum de dor musculoesquelética; sua prevalência é de aproximadamente $47 \%$ na população geral, sendo que entre suas causas, as desordens do manguito rotador (DMR) despontam como as mais frequentes ${ }^{1,2}$. As lesões sintomáticas do manguito rotador (MR) apresentam incidência que varia de $4 \%$ a $32 \%$ sendo mais prevalente com $\circ$ avanço da idade, acometendo mais de $50 \%$ dos indivíduos maiores de 60 anos $^{3,4}$.

As DMR têm importante impacto na saúde e funcionalidade dos seus portadores. Essas lesões limitam a capacidade para a prática de atividades esportivas ou mesmo para realização de atividades de vida diárias (AVD's) que exijam elevação do braço acima do nível do ombro ${ }^{5,6}$. A dor crônica também é um componente constante nos pacientes acometidos por esses distúrbios, causando efeitos físicos e psicossociais. Por todas essas questões sabe-se que as DMR têm um potencial de causar sofrimento humano e perda da qualidade de vida (QV) nos seus portadores ${ }^{1,2,7}$.

Essas desordens são tratadas por técnicas complexas e de longa duração que incluem fisioterapia, medicações e, nos casos mais graves, o tratamento cirúrgico passa a ter papel relevante. Seja qual for o tratamento, os principais objetivos são a eliminação da dor e melhora da funcionalidade do ombro. ${ }^{8,9}$ Independentemente das causas, gravidade ou tipo de tratamento, fica claro que uma avaliação cuidadosa do real impacto das DMR é o ponto chave para uma correta definição diagnóstica e terapêutica. Este tipo de avaliação deveria incluir aspectos subjetivos ligados às expectativas dos sujeitos e não somente avaliar os critérios clínicos e radiológicos tradicionais $^{10}$.

Alguns estudos já tiveram como escopo a análise dos resultados funcionais no tratamento das DMR, contudo poucos trabalhos avaliaram a $Q V$ desses pacientes utilizando um questionário específico para essa doença; mais raros ainda são os estudos que fizeram alguma correlação entre funcionalidade e $Q V^{11,12}$. Questionários genéricos podem ser comumente utilizados para avaliação da $Q V$ em condições ortopédicas, entretanto esses instrumentos genéricos avaliam o impacto global, sendo pobres para mensurar mudanças clínicas resultantes de doenças articulares específicas na QV dos pacientes. ${ }^{13,14}$

Sendo assim, o objetivo deste estudo foi verificar se existe correlação entre qualidade de vida relacionada à saúde e a funcionalidade da articulação do ombro em pacientes portadores de DMR através de questionários específicos para o ombro.

\section{Materiais e métodos}

\section{Desenho do estudo / sujeitos}

Realizou-se estudo do tipo corte transversal com amostra não probabilística do tipo sequencial composta por 109 sujeitos, avaliados no período de outubro de 2013 a fevereiro de 2014 no Serviço de Fisioterapia do Grupo Promédica, Salvador - Bahia - Brasil.

Para finalidade de estimativa do tamanho amostral tomou-se como base o estudo de Kirkley et al. ${ }^{12}$ que estimou a correlação entre WORC com ASES em 0,70 . Desta forma, considerando-se alfa de 0,05 com diferença detectável de $10 \%$, o estudo necessitaria de 81 sujeitos. Acrescentou-se 17 indivíduos ao valor estimado para compensar possíveis perdas. O tamanho amostral estimado ficou, portanto, em um mínimo de 98 indivíduos.

Todos os pacientes que aceitaram participar da pesquisa assinaram o termo de consentimento livre e esclarecido (TCLE) e o projeto de pesquisa foi aprovado pelo Comitê de Ética em Pesquisa da Escola Bahiana de Medicina e Saúde Pública, parecer número 410.457 (CAAE 17940513.1.0000.5544).

\section{Critérios de inclusão}

Pacientes acima de 18 anos de idade que apresentavam diagnóstico clínico de DMR, tais como síndrome do impacto $(\mathrm{SI})$, tendinite calcária ou ruptura do(s) tendão(ões) do $M R$, com mais de 3 meses de sintomatologia no ombro. 


\section{Critérios de exclusão}

Sujeitos com história de osteoartrite radiológica e sintomática das articulações glenoumeral ou acromioclavicular, artrite reumatoide, cervicobraquialgia, desordens cognitivas, doenças neurológicas ou fraturas que acometessem o ombro, capsulite adesi$\mathrm{va}, \mathrm{e}$ aqueles que foram submetidos a algum procedimento cirúrgico no ombro.

\section{Instrumentos/procedimentos}

Os pacientes foram avaliados individualmente, por dois avaliadores treinados e capacitados, em único momento para aplicação dos instrumentos. Foi utilizado questionário sociodemográfico e clínico com os seguintes ítens: nome, idade, sexo, estado civil, dominância, membro(s) acometido(s), membro avaliado, mecanismo da lesão, início dos sintomas, escolaridade, profissão, ocupação, religião, tabagismo, patologia pregressa, atividade física, exames complementares e nome do médico assistente.

Quando a dor apresentava-se bilateralmente, o ombro avaliado foi escolhido pelo paciente como o mais doloroso ou limitado funcionalmente, respeitando os critérios de inclusão. Posteriormente, os pacientes foram orientados a responder de forma autoadministrada os instrumentos UCLA, ASES e WORC para avaliação da funcionalidade e qualidade de vida.

A primeira escala funcional a ser aplicada foi a Modified-University of California at Los Angeles shoulder rating (UCLA-modificada). Esse instrumento apresenta escore com diferentes pesos para os cinco domínios: 1$)$ dor (10 pontos); 2 ) função (10 pontos); 3) flexão anterior ativa (05 pontos); 4) força de flexão anterior ativa (05 pontos) e 5) satisfação do paciente (05 pontos), somando 35 pontos no total. A pontuação foi classificada da seguinte forma: 34-35 pontos correspondem a resultados excelentes, 28-33 bons, 21-27 razoáveis e 0-20 ruins ${ }^{15,16,17}$.

A segunda escala funcional utilizada foi a American Shoulder and Elbow Surgeons Standardized Shoulder Assessment Form (ASES). A ASES apresenta um item relacionado à dor, avaliado por meio de uma EVA $(10 \mathrm{~cm})$ que varia de nenhuma dor $(0)$ à pior dor possível (10), e outro item relacionado à função ${ }^{18,19}$.
Os itens para função foram avaliados por meio de uma escala Likert de quatro pontos com perguntas relacionadas a atividades corriqueiras que fazem parte do cotidiano da maioria das pessoas. Os escores para dor e função foram transformados em porcentagem e cada um representa $50 \%$ do escore final do teste, podendo variar de 0 (nenhuma funcionalidade) a 100 (função normal). 18 Para cálculo da subseção dor multiplicou-se o escore da dor (máximo de 10) por 5, totalizando valor máximo de 50. Para a subseção função realizou-se a soma dos escores da função (máximo de 30 ) e multiplicou-se por $5 / 3$, apresentando valor máximo de 50 . O cálculo final foi tabulado somando-se as subseções dor e função, apresentando valor máximo de $100^{15,16}$.

Para avaliação da qualidade de vida foi aplicado o questionário específico para as DMR, Western Ontario Rotator Cuff Index (WORC). Esse instrumento compreende 21 itens distribuídos em cinco domínios de vida e saúde: 1) Sintomas Físicos (6 itens); 2 ) Esportes/Recreação (4 itens); 3) Trabalho (4 itens); 4) Estilo de Vida (4 itens) e 5) Emoções (3 itens). Para cada item utiliza-se um modelo semelhante à escala visual analógica (EVA), com uma linha medindo $10,0 \mathrm{~cm}$, variando o escore de 0 a $100[11,20]$. A mensuração da distância da extremidade esquerda da linha à marcação realizada pelo paciente foi calculada, apresentando valor máximo de 100 pontos para cada item (registrado até $\circ 0,5 \mathrm{~mm}$ mais próximo), totalizando 2100 pontos para a soma dos cinco domínios. Cada domínio pode ser calculado separadamente, com escore final podendo variar de 0 (sem redução na QV) a 2100 (pior escore possível na $Q V)^{21}$.

Para tornar o resultado final desse instrumento mais compreensível, os autores da versão original do WORC recomendam que os dados sejam convertidos para um escore em porcentagem. Esse valor em pontos, portanto, foi transformado em porcentagem. O mesmo procedimento foi aplicado para cada domínio, com o resultado final expresso em porcentagem variando de 0 (mais baixo nível de QV) a 100 (mais alto nível de $Q V)^{12,15,21}$.

\section{Análise estatística}

Os resultados das variáveis contínuas foram apresentados sob a forma de média \pm desvio padrão ou 
mediana e amplitude interquartil de acordo com a distribuição. As variáveis categóricas foram expressas como proporções. As medidas de tendência central e dispersão foram utilizadas para análise das variáveis quantitativas, e as frequências absoluta e relativa para as qualitativas.

O teste de Spearman foi utilizado para calcular o coeficiente de correlação entre o questionário de $Q V$ e as escalas funcionais. A escolha desse teste baseou-se no fato das variáveis não obedecerem à distribuição normal. Considerou-se as correlações como segue: a) entre 1 e 0,90 (correlação muito alta); b) entre 0,90 e 0,70 (correlação alta); c) entre 0,70 e 0,40 (correlação moderada); d) entre 0,40 e 0,20 (correlação baixa); e) entre 0,20 e 0 (correlação muito baixa) ${ }^{22}$. Para verificação de di- ferenças entre grupos adotou-se em todos os casos o nível de significância de $5 \%(p<0,05)$.

\section{Resultados}

Foram avaliados 109 pacientes, cujo diagnóstico clínico mais frequente foi síndrome do manguito rotador, correspondendo a 72,5\%. Quanto à atividade física, os resultados mostraram que a maioria dos sujeitos não realizava nenhuma atividade física, e a caminhada destacou-se como o exercício mais utilizado entre os praticantes. Detalhes das demais características sociodemográficas podem ser observados na Tabela 1.

Tabela 1. Dados sociodemográficos dos 109 pacientes atendidos no Serviço de Fisioterapia da Promédica de outubro de 2013 a fevereiro de 2014 , Salvador-Bahia

\begin{tabular}{lcc}
\hline \multicolumn{1}{c}{ Variável } & $\mathbf{N}$ & $\%$ \\
\hline Idade (Anos)' $^{\prime}$ & $52,3 \pm 12,8$ & $67,0 \%$ \\
Sexo (Feminino) & 73 & $53,2 \%$ \\
Estado civil (Com companheiro) & 58 & $19,3 \%$ \\
Nível de escolaridade & 21 & $51,4 \%$ \\
$\quad$ Básico & 56 & $29,4 \%$ \\
$\quad$ Médio & 32 & $41,3 \%$ \\
$\quad$ Superior & 45 & \\
Prática de atividade física & & $63,3 \%$ \\
Ocupação & 69 & $10,1 \%$ \\
$\quad$ Trabalha & 11 & $21,1 \%$ \\
$\quad$ Afastado & 23 & $5,5 \%$ \\
$\quad$ Aposentado & 6 & \\
Outro & & \\
\hline
\end{tabular}

1 Representado como média e desvio padrão.

Apesar do ombro direito ter sido o membro mais acometido $(57,8 \%)$, os resultados deste estudo mostraram que a dor bilateral esteve presente em $33,9 \%$ dos sujeitos avaliados. A tabela 2 apresenta as principais características das lesões nos indivíduos avaliados neste estudo. Ao analisar o questionário de QV verificou-se que os domínios "Esportes/Recreação" e "Trabalho" apresentaram as médias mais baixas na QV quando comparados aos demais domínios (Tabela 3). 
Tabela 2. Características da lesão dos 109 pacientes atendidos no Serviço de Fisioterapia da Promédica de outubro de 2013 a fevereiro de 2014 , Salvador-Bahia

\begin{tabular}{|c|c|c|}
\hline $\begin{array}{l}\text { Variável } \\
\end{array}$ & $\mathbf{N}$ & $\%$ \\
\hline Duração dos sintomas (Meses)' & $28,7 \pm 46,1$ & \\
\hline Membro dominante (Direito) & 103 & $94,5 \%$ \\
\hline Membro avaliado (Direito) & 63 & $57,8 \%$ \\
\hline Mecanismo de lesão (Traumático) & 55 & $50,5 \%$ \\
\hline \multicolumn{3}{|l|}{ Exames complementares ${ }^{2}$} \\
\hline \multicolumn{3}{|l|}{ Ultrassom } \\
\hline Ressonância magnética & 68 & $63,5 \%$ \\
\hline \multirow[t]{2}{*}{ Raio-x } & 44 & $41,1 \%$ \\
\hline & 17 & $15,9 \%$ \\
\hline \multicolumn{3}{|l|}{ Tipos de lesão ${ }^{3}$} \\
\hline $\begin{array}{l}\text { Tendinopatia sem } \\
\text { rotura do MR }\end{array}$ & 55 & $51,9 \%$ \\
\hline $\begin{array}{l}\text { Rotura parcial ou } \\
\text { completa do MR }\end{array}$ & 40 & $37,7 \%$ \\
\hline $\begin{array}{l}\text { Calcificação sem rotura } \\
\text { do } M R\end{array}$ & 9 & $8,5 \%$ \\
\hline $\begin{array}{l}\text { Calcificação + Rotura } \\
\text { do MR }\end{array}$ & 2 & $1,9 \%$ \\
\hline
\end{tabular}

Tabela 3. Resultados das escalas aplicadas nos 109 pacientes atendidos no Serviço de Fisioterapia da Promédica de outubro de 2013 a fevereiro de 2014 , Salvador-Bahia

\begin{tabular}{ccc}
\hline Instrumentos de medida & Média & DP \\
\hline Total WORC' (0 - 100) & 47,7 & 27,8 \\
Sintomas Físicos & 56,0 & 27,5 \\
Esportes / Recreação & 40,6 & 29,6 \\
Trabalho & 41,6 & 30,5 \\
Estilo de Vida & 51,5 & 33,0 \\
Emoções & 48,7 & 36,5 \\
CCLA $^{2}(2-35)$ & 20,5 & 7,0 \\
ASES $^{3}(0-100)$ & 52,1 & 20,2 \\
EVA $^{4}(0-10)$ & 5,1 & 2,4
\end{tabular}

1 Western Ontario Rotator Cuff Index / 2 Modified-University of California at Los Angeles Shoulder Rating Scale / ${ }^{3}$ American Shoulder and Elbow Surgeons Scale / ${ }^{4}$ Escala Visual Analógica

Resultados expressos através de média e desvio-padrão.

A qualidade de vida avaliada apresentou correlação positiva alta com as escalas UCLA e ASES. Ao analisar - WORC verificou-se correlação mais forte com a UCLA no domínio "Trabalho" e menor no domínio "Esportes/ Recreação". Quando comparado o WORC com a escala ASES, identificou-se a maior correlação com o domínio "Trabalho" e menor correlação com o domínio "Emoções". A partir dos resultados encontrados foi calculado o coeficiente de correlação entre os instrumentos que avaliaram a QV e a funcionalidade (Tabela 4). 
Tabela 4. Coeficiente de Correlação de Spearman ( $r$ ) entre o WORC, UCLA e ASES dos 109 pacientes atendidos no Serviço de Fisioterapia da Promédica de outubro de 2013 a fevereiro de 2014 , Salvador-Bahia

\begin{tabular}{lcc}
\hline \multicolumn{1}{c}{ WORC } & UCLA & ASES \\
\hline Physical symptoms & 0,71 & 0,76 \\
Sport/Recreation & 0,67 & 0,68 \\
Worc & 0,74 & 0,79 \\
Lifestyle & 0,72 & 0,78 \\
Emotions & 0,68 & 0,64 \\
Total WORC & 0,73 & 0,77 \\
\hline
\end{tabular}

Em todas as correlações encontrou-se um $p<0,001$

\section{Discussão}

Os resultados do nosso estudo evidenciaram correlação significante alta entre a maioria dos domínios da qualidade de vida com a funcionalidade do ombro em pacientes portadores de DMR. Esta correlação foi mais forte para os domínios "Trabalho" ( $r=0,74$ para $\circ$ UCLA e $r=0,79$ para $\circ$ ASES) e "Estilo de Vida" ( $r=0,72$ para o UCLA e $r=0,78$ para ○ ASES), enquanto que o domínio "Emoções" apresentou a correlação mais baixa $(r=0,68$ para - UCLA e $r=0,64$ para o ASES).

Os resultados do nosso trabalho corroboram com outros dois estudos que testaram a correlação entre essas escalas funcionais e o questionário de qualidade de vida WORC ${ }^{17,18}$. Kirkley et al. ${ }^{12}$ testaram - questionário WORC com diversas escalas funcionais e encontraram maior correlação com a ASES $(r=0,76)$ seguida da UCLA que se destacou com correlação alta $(r=0,72)$. Lopes et al. $^{20}$ avaliaram 100 pacientes com diagnóstico de DMR (tendinopatia, lesão do MR e aqueles com mais de 3 meses de cirurgia) e encontraram correlação alta entre a qualidade de vida e a funcionalidade, mensurada pela UCLA $(r=0,80, p<0,001)$. Nosso estudo também apresentou correlação alta entre função e $Q V$, entretanto difere dos autores citados por apresentar análise seccionada correlacionando a funcionalidade (UCLA e ASES) com os diferentes domínios de qualidade de vida do questionário WORC.

Ao analisar o domínio "Trabalho", este se apresentou como a mais forte correlação entre as escalas funcionais e, juntamente com $\circ$ domínio Esportes/Recreação, a média mais baixa entre os domínios da QV. Isso pode ser justificado devido ao fato das DMR apresentarem-se como a segunda maior incidência de dor no ambiente de trabalho, perdendo apenas para patologias na coluna, afetando substancialmente a produtividade no trabalho com indicativo de altas taxas de afastamento e custos atribuídos às lesões do $M R$ no ambiente de trabalho ${ }^{23}$.

Essas desordens também são consideradas como o segundo mais frequente motivo para $\circ$ absenteísmo em trabalhadores manuais, causando grande prejuízo no trabalho, principalmente em atividades que envolvam manipulação frequente de cargas ou com alta força, além de elevação acima do nível do ombro ${ }^{23-24}$. Esses fatores podem justificar $\circ$ grande impacto para esse domínio na população estudada, visto que a maioria dos sujeitos avaliados não estava afastada do trabalho e realizavam atividades manuais, principalmente em ocupações como atividades do lar e professor.

Altas demandas de trabalho estão associadas com a SI, porém a associação entre essa síndrome e fatores psicossociais diferiu em alguns estudos. $O$ que se observa é que a maioria dos trabalhos utiliza o desenho do tipo corte transversal, e esse tipo de estudo não favorece a compreensão dessa associação; desta forma é necessário que outros estudos com desenhos prospectivos sejam realizados para que possam ser confirmadas essa associação ${ }^{24,25}$.

O tratamento inadequado de quadros álgicos em pacientes com dor no ombro pode criar um "ciclo vicioso" de persistente percepção da dor, alterações no padrão do sono, sintomas como depressão e ansiedade, bem como mudanças cognitivas, comportamentais e emocionais ${ }^{26}$. Pesquisadores sugerem que a prevalência de sintomas psicológicos é maior em pacientes com dor crônica do que na população geral e em pacientes com outras patologias sem dor crônica. Cho et al. ${ }^{26}$ realizaram um estudo com 130 pacientes com dor no ombro, 96 desses com diagnóstico de DMR, apresentando dor por no mínimo 3 
meses e tratados conservadoramente. Os resultados desse estudo revelaram prevalência mais alta de sintomas de ansiedade, depressão e alteração do sono em pacientes portadores de DMR evidenciando interferência negativa na qualidade de vida dos pacientes.

No estudo atual, utilizou-se o domínio "Emoções", do questionário de $Q V$, para mensurar estas alterações emocionais e psicológicas. Os resultados confirmam a hipótese de haver correlação entre dor e estado emocional, entretanto o domínio "Emoções" foi - que apresentou correlação menos intensa com a escala ASES e, juntamente com o domínio "Esportes/ Recreação", com a escala UCLA. A força desta correlação pode ter sido subestimada pelo fato das escalas funcionais não apresentarem questões envolvendo aspectos emocionais.

O domínio "Sintomas Físicos" deveria apresentar maior decréscimo da QV por ter maior associação com o sintoma dor. Ao contrário, nos nossos resultados, "Sintomas Físicos" foi a variável com maior média no questionário WORC. Este fato aparentemente se opõe a estudos que apontam que pacientes com DMR tipicamente cursam com dor, fraqueza e limitação de movimento, sendo considerada a dor o seu principal sintoma ${ }^{1,6,27,28}$.

Supõe-se que gravidade e duração dos sintomas esteja relacionada com piora da $Q V$ representando um impacto negativo considerável na funcionalidade dos sujeitos com essas desordens. Contudo, um estudo americano que avaliou 433 indivíduos com lesão completa do $M R$ verificou que não houve correlação entre o tempo de sintomas e os diferentes instrumentos de medida utilizados, dentre eles 0 ASES e WORC, além de nenhuma correlação estatisticamente significante com a gravidade da dor e da lesão do $M R$, força muscular e $A D M^{29}$.

A ausência de estudos na literatura que mostrem correlação estatisticamente significante da dor com a gravidade da lesão do MR pode ser justificada por estudos histológicos que têm revelado que a dor relacionada às DMR parece estar associada com a estimulação das terminações nervosas livres presentes em maior número na bursa, seguida pelo tendão do bíceps e cápsula articular. Apesar dos tendões desempenharem um papel na geração de dor, essas estruturas apresentam poucas terminações nervosas livres o que justifica a hipótese de um mecanismo indireto por alguns peptídeos ou transmissores em iniciar uma resposta à dor pelas fibras da bursa, bíceps ou cápsula articular ${ }^{30}$.

Apesar das causas relacionadas às DMR serem multifatoriais, a prática de esportes e o trabalho envolvendo movimentos repetitivos de elevação do braço acima do nível do ombro, bem como carga excessiva, são citados em diversos estudos como grandes responsáveis pelo surgimento dessas desordens. ${ }^{30-32}$ Neste estudo, foi encontrada alta frequência de sujeitos sedentários e os que praticavam alguma atividade física (caminhada) não realizavam movimentos que exigissem do membro superior. No entanto, há evidências de que o sedentarismo pode contribuir para $\circ$ aparecimento dessas desordens, pois a fraqueza muscular e o desequilíbrio entre as forças combinados (músculos deltoide, MR e estabilizadores escapulares) predispõem ao impacto subacromial com consequente perda funcional ${ }^{3,4,6}$.

desenho do tipo corte transversal foi uma das limitações do estudo, impossibilitando afirmar associação de causa-efeito entre as variáveis. Outra limitação foi a amostragem não probabilística do tipo sequencial, o que pode ter diminuído a validade externa do estudo. A despeito destas limitações, identificar os domínios da QV que apresentam maior correlação com a funcionalidade poderá contribuir para a produção de estudos prospectivos que avaliem os fatores associados e o desenvolvimento de medidas preventivas para essas desordens. Como aspecto forte deste estudo destaca-se a utilização de questionário específico da doença, e escalas funcionais específicas da articulação aplicadas com grande frequência na prática clínica.

\section{Conclusão}

Os autores concluíram que existe correlação estatisticamente significante e alta entre funcionalidade e qualidade de vida de pacientes portadores de DMR. O domínio "Trabalho" destacou-se como a mais forte correlação e o domínio "Emoções" foi o que apresentou a correlação menos intensa. 


\section{Contribuições dos autores}

Albuquerque RA participou da concepção, coleta dos dados, delineamento, busca e análise estatística dos dados da pesquisa, interpretação dos resultados e redação do artigo científico. Castro MMC participou da orientação, análise estatística dos dados da pesquisa, interpretação dos resultados e redação do artigo científico. Matos MA participou da concepção, interpretação dos resultados e redação do artigo científico. Daltro $C$ participou da busca e análise estatística dos dados da pesquisa e interpretação dos resultados.

\section{Conflitos de interesses}

Nenhum conflito financeiro, legal ou político envolvendo terceiros (governo, empresas e fundações privadas, etc.) foi declarado para nenhum aspecto do trabalho submetido (incluindo mas não limitandose a subvenções e financiamentos, conselho consultivo, desenho de estudo, preparação de manuscrito, análise estatística, etc.).

\section{Referências}

1. Gomoll AH, Katz JN, Warner JJ, Millett PJ. Rotator cuff disorders: recognition and management among patients with shoulder pain. Arthritis Rheum. 2004;50(1 2):3751-61. doi: $\underline{10.1002 / \operatorname{art} .20668}$

2. Ekeberg OM, Bautz-Holter E, Juel NG, Engebretsen K, Kvalheim S, Brox Jl. Clinical, socio-demographic and radiological predictors of short-term outcome in rotator cuff disease. BMC Musculoskelet Disord. 2010;1 1:239. doi: 10.1186/1471-2474-11-239

3. Schaer $M$, Schober $M$, Berger S, Boileau $P$, Zumstein MA. Biologically based strategies to augment rotator cuff tears. Int J Shoulder Surg. 201 2;6(2):51-60. doi: 10.4103/0973$\underline{6042.96995}$

4. Van der Meijden OA, Westgard P, Chandler Z, Gaskill TR, Kokmeyer D, Millett PJ. Rehabilitation after arthroscopic rotator cuff repair: current concepts review and evidence based guidelines. Int J Sports Phys Ther. $2012 ; 7(2): 197-218$.

5. Dutton M. Fisioterapia ortopédica: exame, avaliação e intervenção. In: Dutton M. O complexo do ombro. Porto Alegre: Artmed; 2006.

6. Hertling D, Kessler RM. Tratamento de distúrbios musculoesqueléticos comuns: princípios e métodos de fisioterapia. In: Ombro e cintura escapular. Barueri: Manole; 2009.

7. Seitz AL, McClure PW, Finucane S, Boardman ND, Michener LA. Mechanisms of rotator cuff tendinopathy: intrinsic, extrinsic, or both? Clin Biomech. 2011 ;26(1):1-12. doi: 10.1016/i.clinbiomech.2010.08.001
8. Ghodadra NS, Provencher MT, Verma NN, Wilk KE, Romeo AA. Open, mini-open, and all-arthroscopic rotator cuff repair surgery: indications and implications for rehabilitation. J Orthop Sports Phys Ther. 2009;39(2):81-89. doi: 10.2519/ jospt.2009.2918

9. Düzgün I, Baltaci G, Atay OA. Comparison of slow and accelerated rehabilitation protocol after arthroscopic rotator cuff repair: Pain and functional activity. Acta Orthop Traumatol Turc. 201 1;45(1):23-33. doi: 10.3944/ AOTT.2011.2386

10. Puga VOO, Lopes AD, Costa LOP. Assessment of crosscultural adaptations and measurement properties of selfreport outcome measures relevant to shoulder disability in Portuguese: a systematic review. Rev Bras Fisioter. 2012 ; 16(2):85-93.

11. Lopes AD, Stadniky SP, Masiero D, Carrera EF, Ciconelli RM, Griffin S. Tradução e adaptação cultural do WORC: um questionário de qualidade de vida para alterações do manguito Rotador. Rev Bras Fisioter. 2006; 10(3):309-315. doi: $10.1590 /$ S1413-35552006000300010

12. Kirkley A, Alvarez C, Griffin S. The development and evaluation of a disease-specific quality-of-life questionnaire for disorders of the rotator cuff: The Western Ontario Rotator Cuff Index. Clin J Sport Med. 2003;13(2):84-92.

13. Seidl EMF, Zannon CMLC. Qualidade de vida e saúde: aspectos conceituais e metodológicos. Cad Saúde Pública. 2004;20(2):580-88. doi: 10.1590/50102$311 \times 2004000200027$

14. Cho CH, Seo HJ, Bae KC, Lee KJ, Hwang I, Warner JJ. The impact of depression and anxiety on self-assessed pain, disability, and quality of life in patients scheduled for rotator cuff repair. J Shoulder Elbow Surg. 2013;22(9):1 160-6. doi: $10.1016 /$ i.jise.2013.02.006

15. Kirkley A, Griffin S, Dainty K. Scoring systems for the functional assessment of the shoulder. Arthroscopy. 2003;19(10):1 109-20. doi: 10.1016/i.arthro.2003.10.030

16. Wright RW, Baumgarten KM. Shoulder outcomes measures. J Am Acad Orthop Surg. 2010;1 8(7):436-44.

17. Oku EC, Andrade AP, Stadiniky SP, Carrera EF, Tellini GG. Tradução e adaptação cultural do modified-university of California at Los Angeles shoulder rating scale para a Língua Portuguesa. Rev Bras Reumatol. 2006;46(4):246-52. doi: $10.1590 / \mathrm{S} 0482-50042006000400003$
18. Knaut LA, Moser ADL, Melo SA, Richards RR. Tradução e adaptação cultural à língua portuguesa do American Shoulder and Elbow Surgeons Standardized Shoulder Assessment Form (ASES) para avaliação da função do ombro. Rev Bras Reumatol. 2010;50(2):176-89. doi: 10.1590/ $\underline{\mathrm{S} 0482-50042010000200007}$ 
19. Moser ADL, Knaut LAM, Zotz TG, Scharan KO. Validade e confiabilidade da versão em português do American Shoulder and Elbow Surgeons Standardized Shoulder Assessment Form. Rev Bras Reumatol. 201 2;52(3):348-56. doi: $10.1590 /$ S0482-50042012000300005

20. Lopes AD, Ciconelli RM, Carrera EF, Griffin $S$, Faloppa F, Reis FB. Validity and reliability of the Western Ontario Rotator Cuff Index (WORC) for use in Brazil. Clin J Sport Med. 2008; 18(3):266-72. doi: 10.1097/ JSM.Ob013e31817282f4

21. Raman J, MacDermid JC. Western Ontario Rotator Cuff Index. J Physiother. 2012;58(3):201. doi: 10.1016/S18369553(12)70115-7

22. Baquero G. Métodos de pesquisa pedagógica. São Paulo: Edições Loyola; 1973.

23. Kemp KAR, Sheps DM, Luciak-Corea C, Styles-Tripp F, Buckingham J, Beaupre LA. Systematic review of rotator cuff tears in workers' compensation patients. Occup Med. 2011 ;61 (8):556-62. doi: $10.1093 /$ occmed/kgr068

24. Van Rijn RM, Huisstede BMA, Koes BW, Burdorf A. Associations between work-related factors and specific disorders of the shoulder - a systematic review of the literature. Scand J Work Environ Health. 2010;36(3):189201

25. Bodin J, Ha C, Petit Le Manac'h A, Sérazin C, Descatha $A$, Leclerc $A$ et al. Risk factors for incidence of rotator cuff syndrome in a large working population. $2012 ; 38(5): 436-46$. doi: $10.5271 /$ siweh. 3285

26. Cho CH, Jung SW, Park JY, Song KS, Yu KI. Is shoulder pain for three months or longer correlated with depression, anxiety, and sleep disturbance? J Shoulder Elbow Surg. 2013;22(2):222-8. doi: 10.1016/i.jse.2012.04.001

27. DePalma AF. The classic: surgical anatomy of the rotator cuff and the natural history of degenerative periarthritis. Clin Orthop Relat Res. 2008;466(3):543-51. doi: 10.1007/ s1 1999-007-0103-5

28. Heerspink FOL, Hoogeslag RAG, Diercks RL, van Eerden PJM, van den Akker-Scheek, van Raay JJAM. Clinical and radiological outcome of conservative vs. surgical treatment of atraumatic degenerative rotator cuff rupture: design of a randomized controlled trial. BMC Musculoskelet Disord. 2011 ; 12:25. doi: $10.1186 / 1471-2474-12-25$

29. Unruh KP, Kuhn JE, Sanders R, An Q, Baumgarten KM, Bishop JY et al. The duration of symptoms does not correlate with rotator cuff tear severity or other patient-related features: a cross-sectional study of patients with atraumatic, full-thickness rotator cuff tears. J Shoulder Elbow Surg. 2014;23(7):1052-8. doi: 10.1016/i.jse.2013.10.001
30. McFarland EG, Maffulli N, Del Buono A, Murrell GAC, Garzon-Muydi J, Petersen SA. Impingement is not impingement: the case for calling it "Rotator Cuff Disease". Muscles Ligaments Tendons J. 2013;3(3):196-200.

31. Rodgers JA, Crosby LA. Rotator cuff disorders. Am Fam Physician. 1996;54(1):127-34.

32. Metzker CAB. Tratamento conservador na síndrome do impacto no ombro. Fisioter Mov. 2010;23(1):141-51. 